\title{
A PERIODIC SOLUTION OF THE COUPLED MATRIX RICCATI DIFFERENTIAL EQUATIONS
}

\author{
ZAHRA GOODARZI, ABDOLRAHMAN RAZANI, AND M.R. MOKHTARZADEH
}

Received 22 May, 2019

\begin{abstract}
Here, we generalize the martix Riccati differential equation to the coupled matrix Riccati differential equation. Using Schauder's fixed point theorem, the existence of at least one periodic solution of the coupled matrix Riccati equation with $n \times n$ matrix coefficients is proved. Finally, two numerical examples are presented.
\end{abstract}

2010 Mathematics Subject Classification: 34B15; 34C25; 47H10; 54H10

Keywords: coupled matrix Riccati differential equation, Riccati differential equation, periodic solution, compact operator, Schauder's fixed point theorem

\section{INTRODUCTION}

Generally, Riccati differential equation with real valued functions on $\mathbb{R}$ coefficients is $y^{\prime}+p(t) y^{2}+q(t) y=r(t)$. This equation has many applications in different field of sciences such as quantum mechanics in physics, control theory, biomathematics, fluid mechanics, the theory of elastic vibration and econometrics [5, 12,27]. An extensive studies of the set of periodic solutions and nonexistence of periodic solutions are investigated. Also, there are some papers where stability and asymptotic behaviour of solutions were considered. Recently, Ni [17] study the existence and stability of two periodic solutions on a class of Riccati differential equation. Also, Guillot [9], exhibit some families of Riccati differential equations in the complex domain having elliptic coefficients and study the problem of understanding the cases where there are no multi-valued solutions. For more historical background and the existence of solution for this kind of equation see [3, 4, 8, 11, 18].

The term Riccati equation is used to refer to matrix equations with an analogous quadratic term, which occur in both continuous-time and discrete-time linearquadratic-Gaussian control. The steady-state (non-dynamic) version of it is referred to as the algebraic Riccati equation. In a standard manner Riccati equation can be reduced to a second-order linear ordinary differential equations or to a Schrodinger equation of quantum mechanics. In fact, Riccati equation naturally arises in many

The research of A. Razani was in part supported by a grant from IPM (No. 93470122). 
fields of quantum mechanics, in particular, in quantum chemistry, the Wentzel-KramersBrillouin approximation and SUSY theories. Also, methods for solving the GrossPitaevskii equation arising in Bose-Einstein condensates based on Riccati equation are introduced. Hacched et al. [10] consider large scale nonsymmetric differential matrix Riccati equations with low rank right hand sides. These matrix equations appear in many applications such as control theory, transport theory, applied probability and others. Koskela [13] describe the finite dimensional linear quadratic regulator problem. The differential Riccati equation arises in the finite horizon case, i.e., when a finite time integral cost functional is considered. They analysis Krylov subspace approximation to large scale differential Riccati equations. Finally, for more details of application of matrix Riccati equations see [1].

The Riccati differential equation may be generalized as matrix Riccati differential equations (see $[6,7,20]$ ) and it is formulated by $X^{\prime}(t)=A(t) X+X B(t) X+C(t)$, where $A, B$ and $C$ are $n \times n$-real matrix valued function on $\mathbb{R}$. This generalization applies in many areas such as optimal control problem, stochastic control problem and etc. $[2,14,19]$.

In this paper, by Green's function's technique [15, 16,20-26] and Schauder's fixed point theorem, the existence of at least one periodic solution of the coupled matrix Riccati differential equation

$$
\left\{\begin{aligned}
X_{1}^{\prime}(t)= & A(t) X_{1}(t)+X_{1}(t) B_{11}^{(1)}(t) X_{1}(t)+X_{1}(t) B_{12}^{(1)}(t) X_{2}(t) \\
& +X_{2}(t) B_{21}^{(1)}(t) X_{1}(t)+X_{2}(t) B_{22}^{(1)}(t) X_{2}(t)+E_{1}(t), \\
X_{2}^{\prime}(t)= & A(t) X_{2}(t)+X_{1}(t) B_{11}^{(2)}(t) X_{1}(t)+X_{1}(t) B_{12}^{(2)}(t) X_{2}(t) \\
& +X_{2}(t) B_{21}^{(2)}(t) X_{1}(t)+X_{2}(t) B_{22}^{(2)}(t) X_{2}(t)+E_{2}(t),
\end{aligned}\right.
$$

where $A, B_{i j}^{(k)}, E_{k}$ for $i, j, k=1,2$ are $\omega$-periodic continuous matrix valued functions on $\mathbb{R}$, is proved.

In Section 2, by using a suitable Green's function, we can construct a system of integral equation and we can prove the solution of the system of integral equation (2.3) is a solution of the coupled Riccati differential equation (1.1). In Section 3, we construct a compact operator on a Banach space and by applying Schauder's fixed point theorem, it is proved that the system of integral equation (2.3) has at least one periodic solution.

\section{GREEN'S FUNCTION}

Assume $A, B_{i j}^{(k)}, E_{k}$ for $i, j, k=1,2$ are $\omega$-periodic continuous matrix valued functions on $\mathbb{R}$, the coupled matrix Riccati equation (1.1) can be written as 


$$
\left\{\begin{array}{l}
X_{1}^{\prime}=A(t) X_{1}+\sum_{i, j=1}^{2} X_{i}(t) B_{i j}^{(1)}(t) X_{j}(t)+E_{1}(t), \\
X_{2}^{\prime}=A(t) X_{2}+\sum_{i, j=1}^{2} X_{i}(t) B_{i j}^{(2)}(t) X_{j}(t)+E_{2}(t) .
\end{array}\right.
$$

Let $X(t)=\left(X_{1}(t), X_{2}(t)\right)^{T}$, and $\mathbf{B}^{(k)} \in \mathbf{C}\left(\left[0, T_{f}\right], \mathbf{M}_{2 n \times 2 n}(\mathbb{R})\right)$ be $\omega$-periodic real valued matrix functions for $k=1,2$ which are defined by

$$
\mathbf{B}^{(k)}(t)=\left(\begin{array}{cc}
B_{11}^{(k)}(t) & B_{12}^{(k)}(t) \\
B_{21}^{(k)}(t) & B_{22}^{(k)}(t)
\end{array}\right) .
$$

Set $M=\exp \left(\int_{0}^{\omega} A(s) d s\right), M_{1}=\left(I_{n}-M\right)^{-1}$, and $M_{2}=M M_{1}$ where $I_{n}$ is $n \times n$ identity matrix. Notice that $I_{n}-M$ is nonsingular. Define the Green's function $G$ by

$$
G(t, s)= \begin{cases}M_{1} \exp \left(\int_{s}^{t} A(\tau) d \tau\right), & 0 \leq s \leq t \leq \omega, \\ M_{2} \exp \left(\int_{s}^{t} A(\tau) d \tau\right), & 0 \leq t \leq s \leq \omega .\end{cases}
$$

Lemma 1. Suppose $B_{i j}^{(k)}, E_{k}$ for $i, j, k=1,2$ are $\omega$-periodic continuous functions on $\mathbb{R}$. Also, suppose for all $t, s \in[0, \omega], \int_{0}^{t} A(\xi) d \xi$ commutes with $\int_{0}^{s} A(\xi) d \xi$ and $A(t)$, and $A(t)$ commutes with $M$. Let $X$ be a solution of the system of integral equations

$$
\left\{\begin{array}{l}
X_{1}(t)=\int_{0}^{\omega} G(t, s)\left(\sum_{i, j=1}^{2} X_{i}(s) B_{i j}^{(1)}(s) X_{j}(s)+E_{1}(s)\right) d s \\
X_{2}(t)=\int_{0}^{\omega} G(t, s)\left(\sum_{i, j=1}^{2} X_{i}(s) B_{i j}^{(2)}(s) X_{j}(s)+E_{2}(s)\right) d s
\end{array}\right.
$$

then $X(t)$ is a periodic solution of (1.1).

Proof. Since $\int_{0}^{t} A(\xi) d \xi$ commutes with $\int_{0}^{s} A(\xi) d \xi$, one may write

$$
\left(\int_{0}^{t} A(\xi) d \xi\right)\left(-\int_{0}^{s} A(\xi) d \xi\right)=\left(-\int_{0}^{s} A(\xi) d \xi\right)\left(\int_{0}^{t} A(\xi) d \xi\right) .
$$

Therefore

$$
\begin{aligned}
\exp \left(\int_{s}^{t} A(\xi) d \xi\right) & =\exp \left(\int_{0}^{t} A(\xi) d \xi-\int_{0}^{s} A(\xi) d \xi\right)=\exp \left(\int_{0}^{t} A(\xi) d \xi\right) \exp \left(-\int_{0}^{s} A(\xi) d \xi\right) \\
& =\exp \left(\int_{0}^{t} A(\xi) d \xi\right)\left(\exp \left(\int_{0}^{s} A(\xi) d \xi\right)\right)^{-1}
\end{aligned}
$$


Set $\alpha(t):=\exp \left(\int_{0}^{t} A(\xi) d \xi\right)$, thus

$$
\begin{aligned}
X_{1}(t)= & \int_{0}^{\omega} G(t, s)\left(\sum_{i, j=1}^{2} X_{i}(s) B_{i j}^{(1)}(s) X_{j}(s)+E_{1}(s)\right) d s \\
= & \int_{0}^{t} G(t, s)\left(\sum_{i, j=1}^{2} X_{i}(s) B_{i j}^{(1)}(s) X_{j}(s)+E_{1}(s)\right) d s \\
& +\int_{t}^{\omega} G(t, s)\left(\sum_{i, j=1}^{2} X_{i}(s) B_{i j}^{(1)}(s) X_{j}(s)+E_{1}(s)\right) d s \\
= & M_{1} \int_{0}^{t} \exp \left(\int_{s}^{t} A(\xi) d \xi\right)\left(\sum_{i, j=1}^{2} X_{i}(s) B_{i j}^{(1)}(s) X_{j}(s)+E_{1}(s)\right) d s \\
& -M_{2} \int_{\omega}^{t} \exp \left(\int_{s}^{t} A(\xi) d \xi\right)\left(\sum_{i, j=1}^{2} X_{i}(s) B_{i j}^{(1)}(s) X_{j}(s)+E_{1}(s)\right) d s \\
= & M_{1} \alpha(t) \int_{0}^{t} \alpha(s)^{-1}\left(\sum_{i, j=1}^{2} X_{i}(s) B_{i j}^{(1)}(s) X_{j}(s)+E_{1}(s)\right) d s \\
& -M_{2} \alpha(t) \int_{\omega}^{t} \alpha(s)^{-1}\left(\sum_{i, j=1}^{2} X_{i}(s) B_{i j}^{(1)}(s) X_{j}(s)+E_{1}(s)\right) d s .
\end{aligned}
$$

Since $\int_{0}^{t} A(\xi) d \xi$ commutes with $A(t)$, the chain rule is satisfied and

$$
\begin{aligned}
X_{1}^{\prime}(t)= & M_{1} A(t) \alpha(t) \int_{0}^{t} \alpha(s)^{-1}\left(\sum_{i, j=1}^{2} X_{i}(s) B_{i j}^{(1)}(s) X_{j}(s)+E_{1}(s)\right) d s \\
& +M_{1}\left(\sum_{i, j=1}^{2} X_{i}(t) B_{i j}^{(1)}(t) X_{j}(t)+E_{1}(t)\right) \\
& -M_{2} A(t) \alpha(t) \int_{\omega}^{t} \alpha(s)^{-1}\left(\sum_{i, j=1}^{2} X_{i}(s) B_{i j}^{(1)}(s) X_{j}(s)+E_{1}(s)\right) d s \\
& -M_{2}\left(\sum_{i, j=1}^{2} X_{i}(t) B_{i j}^{(1)}(t) X_{j}(t)+E_{1}(t)\right) .
\end{aligned}
$$


Therefore

$$
\begin{aligned}
X_{1}^{\prime}(t)= & A(t) \int_{0}^{t} M_{1} \exp \left(\int_{s}^{t} A(\xi) d \xi\right)\left(\sum_{i, j=1}^{2} X_{i}(s) B_{i j}^{(1)}(s) X_{j}(s)+E_{1}(s)\right) d s \\
& +A(t) \int_{t}^{\omega} M_{2} \exp \left(\int_{s}^{t} A(\xi) d \xi\right)\left(\sum_{i, j=1}^{2} X_{i}(s) B_{i j}^{(1)}(s) X_{j}(s)+E_{1}(s)\right) d s \\
& +\left(M_{1}-M_{2}\right)\left(\sum_{i, j=1}^{2} X_{i}(t) B_{i j}^{(1)}(t) X_{j}(t)+E_{1}(t)\right) \\
= & A(t) \int_{0}^{t} G(t, s)\left(\sum_{i, j=1}^{2} X_{i}(s) B_{i j}^{(1)}(s) X_{j}(s)+E_{1}(s)\right) d s \\
& +A(t) \int_{t}^{\omega} G(t, s)\left(\sum_{i, j=1}^{2} X_{i}(s) B_{i j}^{(1)}(s) X_{j}(s)+E_{1}(s)\right) d s \\
& +\sum_{i, j=1}^{2} X_{i}(t) B_{i j}^{(1)}(t) X_{j}(t)+E_{1}(t) \\
= & A(t) \int_{0}^{\omega} G(t, s)\left(\sum_{i, j=1}^{2} X_{i}(s) B_{i j}^{(1)}(s) X_{j}(s)+E_{1}(s)\right) d s \\
& +\sum_{i, j=1}^{2} X_{i}(t) B_{i j}^{(1)}(t) X_{j}(t)+E_{1}(t) \\
= & A(t) X_{1}(t)+\sum_{i, j=1}^{2} X_{i}(t) B_{i j}^{(1)}(t) X_{j}(t)+E_{1}(t) .
\end{aligned}
$$

With the similar method, we have the same for $X_{2}(t)$. So, we conclude that $X(t)$ is a solution of equation (1.1).

\section{PERIOdiC SOlUtion}

Here, we prove the existence of at least one periodic solution of the system (1.1).

Theorem 1. Suppose the coefficients of the system (1.1) are $\omega$-periodic continuous functions on $\mathbb{R}$ and the assumptions of Lemma 1 are are satisfied. For $j=1,2$, set

$$
\mu=\sup _{0 \leq t, s \leq \omega}\|G(t, s)\|, v=\max _{0 \leq t \leq \omega, j=1,2}\left\|\int_{0}^{\omega} G(t, s) E_{j}(s) d s\right\| .
$$


If

$$
\max \left\{\int_{0}^{\omega} \| B^{(k)}|| d s \mid k=1,2\right\} \leq \frac{1}{4 \mu \nu},
$$

where $\left\|\boldsymbol{B}^{(k)}\right\|$ is operator norm of $2 n \times 2 n$ matrix $\boldsymbol{B}^{(k)}$. Then system (1.1) admits at least one $\omega$-periodic solution. $\bar{R}$.

Remark 1. For convenience, set $\underline{R}=\max \left\{\int_{0}^{\omega} \| B_{i j}^{(k)}|| d s \mid i, j, k=1,2\right\}$ and $\frac{1}{4 \mu \nu}=$ Proof. Suppose $\phi(t)=\left(\phi_{1}(t), \phi_{2}(t)\right)^{T}$ and

$\mathbf{X}=\left\{\phi(t) \mid \phi_{1}(t), \phi_{2}(t)\right.$ are $\omega$-periodic continuous functions from $\mathbb{R}$ to $\left.\mathbb{M}_{n}(\mathbb{R})\right\}$,

which is equipped with the norm $\|\phi\|_{\mathbf{X}}=\max _{j=1,2}\left\|\phi_{j}\right\|_{\omega}$, where $\left\|\phi_{j}\right\|_{\omega}$ is operator matrix norm for $n \times n$ matrices. $\left(X,\|\cdot\|_{X}\right)$ is a Banach space. Set $\psi(t)=$ $\left(\int_{0}^{\omega} G(t, s) E_{1}(s) d s, \int_{0}^{\omega} G(t, s) E_{2}(s) d s\right)^{T}$ and define a set $\mathbf{F}=\left\{\phi \in \mathbf{X} \mid\|\phi-\psi\|_{\mathbf{X}} \leq\right.$ $\nu$. Notice that $\mathbf{F}$ is closed, bounded and convex subset of $\mathbf{X}$. Define $P: \mathbf{F} \rightarrow \mathbf{X}$ by

$$
P(\phi)(t)=\left(\begin{array}{c}
\int_{0}^{\omega} G(t, s) \sum_{i, j=1}^{2} \phi_{i}(s) B_{i j}^{(1)} \phi_{j}(s)+E_{1}(s) d s \\
\int_{0}^{\omega} G(t, s) \sum_{i, j=1}^{2} \phi_{i}(s) B_{i j}^{(2)} \phi_{j}(s)+E_{2}(s) d s
\end{array}\right) .
$$

It's easy to see that $\|\phi(t)\|_{\mathbf{X}} \leq v+\|\psi(t)\|_{\mathbf{X}} \leq 2 v$ for all $t \in[0, \omega]$. By using the sub multiplicative property of the operator norm, for all $t \in[0, \omega]$ we have

$$
\begin{aligned}
\|P(\phi)(t)-\psi(t)\| \mathbf{x} & \leq\left\|\int_{0}^{\omega} G(t, s) \phi^{T} \mathbf{B}^{(k)} \phi d s\right\| \\
& \leq \int_{0}^{\omega}\left\|G(t, s) \phi^{T}(s) \mathbf{B}^{(k)}(s) \phi(s)\right\| d s \\
& \leq \int_{0}^{\omega}\|G(t, s)\|\left\|\mathbf{B}^{(k)}(s)\right\|\|\phi(s)\|^{2} d s \\
& \leq 4 \mu v^{2} \int_{0}^{\omega}\left\|\mathbf{B}^{k}\right\| d s \\
& \leq v .
\end{aligned}
$$

Thus for all $\phi \in \mathbf{F}$ we have $\|P(\phi)-\psi\|_{\mathbf{x}} \leq v$ and so $P(\phi) \in \mathbf{F}$. This shows $P$ is an operator from $\mathbf{F}$ into $\mathbf{F}$.

Now, we recall the weak version of Ascoli-Arzelà theorem to prove the compactness of $P$.

Lemma 2 (Ascoli - Arzela). Let $\left\{\Phi_{n}(t)\right\}_{n \in \mathbb{N}}$ be a sequence of functions from $[a, b]$ to $\mathbb{R}^{2}$ which is uniformly bounded and equicontinuous. Then $\left\{\Phi_{n}(t)\right\}_{n \in \mathbb{N}}$ has a uniformly convergent subsequence. 
Suppose $\left\{\phi_{n}\right\}=\left(\left\{\left(\phi_{1}\right)_{n}\right\},\left\{\left(\phi_{2}\right)_{n}\right\}\right)^{T}$, where ${ }^{T}$ means transpose, is a sequence on F. This sequence is bounded. So, there exists $\lambda>0$ such that for all $n \in \mathbb{N}$ and for all $t \in[0, \omega]$, we have $\left\|\phi_{n}(t)\right\|_{\mathbf{x}} \leq \lambda$. At following, we have to show $\left\{\phi_{n}\right\}$ has a subsequence, $\left\{\phi_{n_{i}}\right\}$, such that $\left\{P\left(\phi_{n_{i}}\right)\right\}$ is convergent on $\mathbf{F}$.

According to Lemma 1 , the function $P\left(\phi_{n}\right)$ is differentiable and for all $t \in[0, \omega]$, we have

$$
P\left(\phi_{n}\right)^{\prime}(t)=\left(\begin{array}{c}
A(t)\left(\phi_{1}\right)_{n}(t)+\sum_{i, j=1}^{2}\left(\phi_{i}\right)_{n}(t) B_{i j}^{(1)}\left(\phi_{j}\right)_{n}(t)+E_{1}(t) \\
A(t)\left(\phi_{2}\right)_{n}(t)+\sum_{i, j=1}^{2}\left(\phi_{i}\right)_{n}(t) B_{i j}^{(2)}\left(\phi_{j}\right)_{n}(t)+E_{2}(t)
\end{array}\right) .
$$

Since $\mathbf{F}$ is bounded for all $n \in \mathbb{N}$ and for all $t \in[0, \omega]$, we get $\left\|P\left(\phi_{n}\right)^{\prime}(t)\right\|_{\mathbf{X}} \leq \rho_{1} \lambda+$ $\rho_{2} \lambda^{2}+\rho_{3}$, where $\rho_{1}, \rho_{2}$ and $\rho_{3}$ are $\|A\|_{\omega}, \max \left\{\left\|B^{(k)}\right\| \| k=1,2,0 \leq t \leq \omega\right\}$ and $\max _{k=1,2}\left\|E_{k}\right\|_{\omega}$ on $[0, \omega]$, respectively. For given $\varepsilon>0$, let $\delta=\varepsilon /\left(\rho_{1} \lambda+\rho_{2} \lambda^{2}+\right.$ $\left.\rho_{3}\right)$, then for all $n \in \mathbb{N}$ and for all $t_{1}, t_{2} \in[0, \omega],\left|t_{1}-t_{2}\right|<\delta$ implies that

$$
\left\|P\left(\phi_{n}\right)\left(t_{1}\right)-P\left(\phi_{n}\right)\left(t_{2}\right)\right\| \mathbf{x} \leq\left(\rho_{1} \lambda+\rho_{2} \lambda^{2}+\rho_{3}\right)\left|t_{1}-t_{2}\right|<\varepsilon .
$$

So $\left\{P\left(\phi_{n}(t)\right)\right\}$ is equicontinuous and Theorem 2 implies that there exists a subsequence of $\left\{P\left(\phi_{n_{i}}(t)\right)\right\}$ of $\left\{P\left(\phi_{n}(t)\right)\right\}$ which is uniformly convergent on $[0, \omega]$. We conclude that $\left\{P\left(\phi_{n_{i}}\right)\right\}$ is convergent on $\mathbf{F}$ and so $P$ is compact.

Thus Schauder's fixed point theorem implies there exists $X(t)=\left(X_{1}(t), X_{2}(t)\right)^{T} \in \mathbf{F}$ such that $P(X(t))=X(t)$, i.e. for all $t \in[0, \omega]$

$$
X(t)=\left(\begin{array}{c}
X_{1}(t) \\
X_{2}(t)
\end{array}\right)=\left(\begin{array}{c}
\int_{0}^{\omega} G(t, s)\left(\sum_{i, j=1}^{2} X_{i}(s) B_{i j}^{(1)} X_{j}(s)+E_{1}(s)\right) d s \\
\int_{0}^{\omega} G(t, s)\left(\sum_{i, j=1}^{2} X_{i}(s) B_{i j}^{(2)} X_{j}(s)+E_{2}(s)\right) d s
\end{array}\right) .
$$

Thus, by Lemma 1, $X(t)$ is a solution of equation (1.1).

Before ending this section, we would like to generalize the system (2.1).

Remark 2. Notice that the developments in this paper may be done in the following more general case

$$
\left\{\begin{array}{l}
X_{1}^{\prime}=A_{1}(t) X_{1}+X_{1} D_{1}(t)+\sum_{i, j=1}^{2} X_{i}(t) B_{i j}^{(1)}(t) X_{j}(t)+E_{1}(t), \\
X_{2}^{\prime}=A_{2}(t) X_{2}+X_{2} D_{2}(t)+\sum_{i, j=1}^{2} X_{i}(t) B_{i j}^{(2)}(t) X_{j}(t)+E_{2}(t),
\end{array}\right.
$$

where $A_{k}, D_{k}, B_{i j}^{(k)}, E_{k}$ for $i, j, k=1,2$ are $\omega$-periodic continuous matrix valued functions on $\mathbb{R}$. A good question is the existence of at least one period solution of (3.4).

Note that the system (3.4) is the system (2.1) when $D_{1}(t)=D_{2}(t)=0$ and $A_{1}(t)=A_{2}(t)=A(t)$. 


\section{NUMERICAL EXAMPLES}

In this section, we bring two numerical examples that show the novelty of the results.

Example 1. Consider the family of the coupled matrix Riccati equation (2.3) with the coefficients

$$
\begin{aligned}
& A(t)=\left(\begin{array}{ll}
\frac{3}{16} & \frac{-1}{16} \\
\frac{1}{16} & \frac{1}{16}
\end{array}\right), \\
& B_{11}^{1}(t)=\left(\begin{array}{cc}
\frac{\cos t}{4} & \frac{\sin t}{4} \\
\frac{-\sin t}{4} & \frac{\cos t}{4}
\end{array}\right), \quad B_{12}^{1}(t)=\left(\begin{array}{ll}
0 & 0 \\
0 & 0
\end{array}\right), \\
& B_{21}^{1}(t)=\left(\begin{array}{ll}
0 & 0 \\
0 & 0
\end{array}\right), \quad B_{22}^{1}(t)=\left(\begin{array}{cc}
\frac{\cos t}{4} & \frac{-\sin t}{4} \\
\frac{\sin t}{4} & \frac{\cos t}{4}
\end{array}\right), \\
& B_{11}^{2}(t)=\left(\begin{array}{cc}
\frac{\cos t}{4} & \frac{\sin t}{4} \\
\frac{-\sin t}{4} & \frac{\cos t}{4}
\end{array}\right), \quad B_{12}^{2}(t)=\left(\begin{array}{ll}
0 & 0 \\
0 & 0
\end{array}\right), \\
& B_{21}^{2}(t)=\left(\begin{array}{ll}
0 & 0 \\
0 & 0
\end{array}\right), \quad B_{22}^{1}(t)=\left(\begin{array}{cc}
\frac{\cos t}{4} & \frac{-\sin t}{4} \\
\frac{\sin t}{4} & \frac{\cos t}{4}
\end{array}\right) \text {, } \\
& E_{1}(t)=\left(\begin{array}{cc}
\frac{511 \cos t-65 \sin t}{4096} & \frac{-3(32 \cos t+171 \sin t)}{4096} \\
\frac{1022 \cos t-129 \sin t+\sin 3 t}{5192} & \frac{-64 \cos t-\sin t-\sin 3 t}{5192}
\end{array}\right),
\end{aligned}
$$

and

$$
E_{2}(t)=\left(\begin{array}{cc}
\frac{-8257 \cos t-63 \cos 3 t-65599 \sin t-65 \sin 3 t}{524288} & \frac{65536 \cos t-122225 \sin t-65 \sin 3 t}{524288} \\
\frac{-16577 \cos t-63 \cos 3 t-131133 \sin t-65 \sin 3 t}{1048576} & \frac{-8257 \sin t-65 \sin 3 t}{1048576}
\end{array}\right) .
$$

Then

$$
M=\left(\begin{array}{cc}
\frac{1}{8} \exp \pi / 4(8+\pi) & \frac{-1}{8} \exp \pi / 4 \pi \\
\frac{-1}{8} \exp \pi / 4 \pi & \frac{-1}{8} \exp \pi / 4(-8+\pi)
\end{array}\right) .
$$

Therefore, $\underline{R}<\bar{R}$ and the equation (1.1) has a periodic solution.

Remark 3. We can easily show that

$$
X_{1}=\left(\begin{array}{cc}
\frac{\sin t}{8} & \frac{\cos t}{8} \\
\frac{\sin t}{8} & 0
\end{array}\right) \text { and } X_{2}=\left(\begin{array}{cc}
\frac{\cos t}{8} & \frac{\sin t}{8} \\
\frac{\sin t}{8} & 0
\end{array}\right)
$$

is a periodic solution whose existence is guaranteed.

Remark 4. Consider the initial value problem consist of the Riccati equation in example 1 together with initial condition $X(0)=X_{0}$. Let

$$
\left\{\begin{array}{l}
T_{1} X(t)=\int_{0}^{t}\left[A(s) X_{1}(s)+\sum_{i, j=1}^{2} X_{i}(s) B_{i j}^{(1)}(s) X_{j}(s)+E_{1}(s)\right] d s+\xi_{1}, \\
T_{2} X(t)=\int_{0}^{t}\left[A(s) X_{2}(s)+\sum_{i, j=1}^{2} X_{i}(s) B_{i j}^{(2)}(s) X_{j}(s)+E_{2}(s)\right] d s+\xi_{2},
\end{array}\right.
$$


then $T: \mathbf{C}\left(\mathbb{R}, \mathbf{M}_{2 \times 2}(\mathbb{R})\right) \times \mathbf{C}\left(\mathbb{R}, \mathbf{M}_{2 \times 2}(\mathbb{R})\right) \rightarrow \mathbf{C}\left(\mathbb{R}, \mathbf{M}_{2 \times 2}(\mathbb{R})\right) \times \mathbf{C}\left(\mathbb{R}, \mathbf{M}_{2 \times 2}(\mathbb{R})\right)$, where $T=\left(T_{1}, T_{2},\right)^{T}$ is the corresponding Picard's operator that relates the unique solution of the initial value problem to a fixed point of an operator. The fixed point iteration method

$$
\left\{\begin{array}{l}
X_{n}(t)=T X_{n-1}(t) \\
X_{0}(t)=\xi
\end{array}\right.
$$

where $\xi=\left(\xi_{1}, \xi_{2}\right)^{T}$ constitutes a numerical technique for generating the unique solution of the initial value problem, provided that the convergence is guaranteed. Let $E R_{n}=\left\|X_{n}-X\right\|=\max _{0 \leq t \leq 2 \pi}\left\|X_{n}(t)-X(t)\right\|$, be the error of approximation, where

where

$$
X_{1}=\left(\begin{array}{cc}
\frac{\sin t}{8} & \frac{\cos t}{8} \\
\frac{\sin t}{8} & 0
\end{array}\right) \text { and } X_{2}=\left(\begin{array}{cc}
\frac{\cos t}{8} & \frac{\sin t}{8} \\
\frac{\sin t}{8} & 0
\end{array}\right)
$$

$$
\xi_{1}=\left(\begin{array}{cc}
0 & \frac{1}{8} \\
0 & 0
\end{array}\right) \text { and } \xi_{2}=\left(\begin{array}{cc}
\frac{1}{8} & 0 \\
\frac{1}{8} & 0
\end{array}\right) \text {. }
$$

Numerical values for the error are given in the Table 1.

TABLE 1.

\begin{tabular}{|c|c|c|}
\hline$n$ & $E R_{1}$ & $E R_{2}$ \\
\hline 1 & 0.19635 & 0.19635 \\
\hline 2 & 0.11539 & 0.0774063 \\
\hline 3 & 0.0336085 & 0.0190035 \\
\hline 4 & 0.00657322 & 0.00521541 \\
\hline 5 & 0.000982148 & 0.000993719 \\
\hline 6 & 0.000118411 & 0.000147843 \\
\hline 7 & 0.0000203268 & 0.0000202704 \\
\hline 8 & $3.00827 \times 10^{-} 6$ & $2017528 \times 10^{-} 6$ \\
\hline
\end{tabular}

Example 2. Consider the Riccati equation (1.1) with coefficients

$$
\begin{aligned}
& A(t)=\left(\begin{array}{cc}
\frac{1}{5}(1+\cos t) & \frac{1}{5}(1+\sin t) \\
\frac{1}{5}(-1-\sin t) & \frac{1}{5}(1+\cos t)
\end{array}\right), \\
& B_{11}^{1}(t)=\left(\begin{array}{cc}
\frac{\cos t}{25} & \frac{\sin t}{25} \\
\frac{-\sin t}{25} & \frac{\cos t}{25}
\end{array}\right) \text {, } \\
& B_{21}^{1}(t)=\left(\begin{array}{cc}
0 & 0 \\
0 & 0
\end{array}\right) \text {, } \\
& B_{11}^{2}(t)=\left(\begin{array}{cc}
\frac{\cos t}{25} & \frac{\sin t}{25} \\
\frac{-\sin t}{25} & \frac{\cos t}{25}
\end{array}\right), \\
& B_{21}^{2}(t)=\left(\begin{array}{cc}
0 & 0 \\
0 & 0
\end{array}\right) \text {, } \\
& B_{12}^{1}(t)=\left(\begin{array}{cc}
\frac{1}{25} & \frac{1}{25} \\
\frac{-1}{25} & \frac{1}{25}
\end{array}\right), \\
& B_{22}^{1}(t)=\left(\begin{array}{cc}
0 & 0 \\
0 & 0
\end{array}\right)^{25} \text {, } \\
& B_{12}^{2}(t)=\left(\begin{array}{ll}
\frac{1}{25} & \frac{1}{25} \\
\frac{-1}{25} & \frac{1}{25}
\end{array}\right), \\
& B_{22}^{2}(t)=\left(\begin{array}{cc}
0 & 0 \\
0 & 0
\end{array}\right)^{25} \text {, }
\end{aligned}
$$




$$
E_{1}=\left(\begin{array}{cc}
\frac{1-25 \cos t-25 \cos 2 t-\cos 3 t-100 \sin t}{625} & \frac{-1-25 \cos t-100 \cos t-25 \sin t-25 \sin 2 t-\sin 3 t}{625} \\
\frac{1+25 \cos t+100 \cos t+25 \sin t+25 \sin 2 t+\sin 3 t}{625} & \frac{1-25 \cos t-25 \cos 2 t-\cos 3 t-100 \sin t}{625}
\end{array}\right)
$$

and

$$
E_{2}=\left(\begin{array}{ll}
\frac{1-26 \cos t-25 \cos 2 t-\cos 3 t-150 \sin t}{625} & \frac{-1-25 \cos t-150 \cos t+25 \sin t+\sin 3 t}{625} \\
\frac{1+25 \cos t+150 \cos t-25 \sin t-\sin 3 t}{625} & \frac{1-26 \cos t-25 \cos 2 t-\cos 3 t-150 \sin t}{625}
\end{array}\right) .
$$

Then

$$
M=\left(\begin{array}{cc}
\frac{1}{4}(-1+\sqrt{5}) \exp 2 \pi / 5 & \sqrt{\frac{5}{8}+\frac{\sqrt{5}}{8}} \exp 2 \pi / 5 \\
\sqrt{\frac{5}{8}+\frac{\sqrt{5}}{8}} \exp 2 \pi / 5 & \frac{1}{4}(-1+\sqrt{5}) \exp 2 \pi / 5
\end{array}\right)
$$

Remark 5. For consistency of our assumptions, we can easily show that

$$
X_{1}=\left(\begin{array}{cc}
\frac{\cos t}{5} & \frac{\cos t}{5} \\
\frac{-\cos t}{5} & \frac{\cos t}{5}
\end{array}\right) \text { and } X_{2}=\left(\begin{array}{cc}
\frac{\cos t}{5} & \frac{-\cos t}{5} \\
\frac{\cos t}{5} & \frac{\cos t}{5}
\end{array}\right)
$$

is a periodic solution. Consider the fixed point iteration corresponding to this example (see example (1)). Numerical results are shown in the Table 2.

TABLE 2.

\begin{tabular}{|c|c|c|}
\hline$n$ & $E_{1}$ & $E_{2}$ \\
\hline 1 & 0.502655 & 0.389338 \\
\hline 2 & 0.403075 & 0.380026 \\
\hline 3 & 0.176354 & 0.184883 \\
\hline 4 & 0.0797213 & 0.0770404 \\
\hline 5 & 0.0180976 & 0.0244803 \\
\hline 6 & 0.00618601 & 0.00610193 \\
\hline 7 & 0.00118407 & 0.0013605 \\
\hline 8 & 0.000367802 & 0.000436594 \\
\hline 9 & 0.000219501 & 0.0001916484 \\
\hline 10 & 0.0000643376 & 0.0000556998 \\
\hline 11 & 0.0000296998 & $5.65144 \times 10^{-6}$ \\
\hline 12 & $4.17076 \times 10^{-} 6$ & 0.0000127965 \\
\hline
\end{tabular}

\section{REFERENCES}

[1] H. Abou-Kandil, G. Freiling, V. Ionescu, and G. Jank, Matrix Riccati Equations in Control and Systems Theory. Birkhäuser, Basel, 2003.

[2] S. Bittanti, P. Colaneri, and G. D. Nicolao, "The periodic riccati equation," Comm. Control Engrg. Ser., pp. 127-162, 1991.

[3] S. Bittanti, P. Colaneri, and G. D. Nicolao, "Special solutions of bi-riccati delay-differential equations," Symmetry, Integrability and Geometry: Methods and Applications, vol. 14, no. 020, p. 9, 2018 . 
[4] S. Castillo and M. Pinto, "Existence and stability of almost periodic solutions to differential equations with piecewise constant arguments," Electronic Journal of Differential Equations, vol. 2015, no. 58 , pp. 1-15, 2015.

[5] E. Fraga, The Schrödinger and Riccati Equations. Springer-Verlag, 1999, vol. 70.

[6] Z. Goodarzi and A. Razani, An application of Green's function. International Congress of Mathematicians 2014 (ICM2014), Seoul, South Korea, 2014.

[7] Z. Goodarzi and A. Razani, "A periodic solution of the generalized forced liénard equation," $A b$ stract and Applied Analysis, vol. 2014, no. 132450, p. 5 pages, 2014.

[8] K. Y. Guan, J. Gunson, and H. S. Hassan, "On periodic solutions of the periodic riccati equation," Results in Mathematics, vol. 14, no. 3-4, pp. 309-317, 1998.

[9] A. Guillot, "Further riccati differential equations with elliptic coefficients and meromorphic solutions," Journal Journal of Nonlinear Mathematical Physics, vol. 25, no. 3, pp. 497-508, 2018.

[10] M. Hacched and K. Jbilou, "Approximate solutions to large nonsymmetric differential riccati problem," arXiv:1801.01291v1, p. 11, 2018.

[11] M. Jungers, "Historical perspectives of the riccati equations," IFAC PapersOnLine, vol. 50, no. 1, pp. 9535-9546, 2017.

[12] A. Khare and U. Sukhatme, Supersymmetry in Quantum Mechanics. World Scientific, Singapore, 2001.

[13] A. Koskela and H. Mena, "Analysis of krylov subspace approximation to large scale differential riccati equations," arXiv:1705.07507v3, p. 24, 2018.

[14] W. M. McEneaney, "A new fundamental solution for differential riccati equations arising in control," Automatica, vol. 44, pp. 920-936, 2008.

[15] M. Mokhtarzadeh, M. R. Pournaki, and A. Razani, "A note on periodic soultions of riccati equations," Nonlinear Dynamics, vol. 62, no. 1-2, pp. 119-125, 2010.

[16] M. Mokhtarzadeh, M. R. Pournaki, and A. Razani, "An existence-uniqueness theorem for a class of boundary value problems," Fixed Point Theory, vol. 13, no. 2, pp. 583-592, 2012.

[17] H. Ni, "The existence and stability of two periodic solutions on a class of riccati's equation," Mathematical Problems in Engineering, vol. 2805161, p. 18, 2018.

[18] Y. Pala and M. Ertas, "A new analytical method for solving general riccati equation," Universal Journal of Applied Mathematics, vol. 5, no. 2, pp. 11-16, 2017.

[19] G. P. Papavassilopoulos and J. Cruz, "On the existence of solutions of coupled matrix riccati differential equations in linear quadratic nash games," IEEE Transactions on Automatic Control, vol. 24, no. 1, pp. 127-129, 1979.

[20] M. R. Pournaki and A. Razani, "On the existence of periodic solutions for a class of generalized forced liénard equations," Applied Mathematics letters, vol. 20, pp. 248-254, 2007.

[21] A. Razani, "Shock waves in gas dynamics," Surveys in Mathematics and its Applications, vol. 2, pp. 59-89, 2007.

[22] A. Razani, Results in Fixed Point Theory. Andisheh Zarin publisher, 2010.

[23] A. Razani, "An existence theorem for ordinary differential equation in menger probabilistic metric space," Miskolc Mathematical Notes, vol. 15, no. 2, pp. 711-716, 2014.

[24] A. Razani, "Chapman-jouguet travelling wave for a two-steps reaction scheme," Italian Journal of Pure and Applied Mathematics, vol. 39, pp. 544-553, 2018.

[25] A. Razani and Z. Goodarzi, "A solution of volterra-hamerstain integral equation in partially ordered sets," Int. J. Industrial Mathematics, vol. 3, no. 4, pp. 277-281, 2011.

[26] A. Razani and Z. Goodarzi, "Iteration by cesàro means for quasi-contractive mappings," Filomat, vol. 28, no. 8, pp. 1575-1584, 2014.

[27] M. Shayman, "On the phase portrait of the matrix riccati equation arising from the periodic control problem,” SIAM J. Control Optim., vol. 23, no. 5, pp. 717-751, 1985. 
Authors' addresses

\section{Zahra Goodarzi}

Imam Khomeini International University, Faculty of Science, Department of Pure Mathematics, Postal code: 34149-16818, Qazvin, Iran

E-mail address: z.goodarzi@edu.ikiu.ac.ir

\section{Abdolrahman Razani}

Imam Khomeini International University, Faculty of Science, Department of Pure Mathematics, Postal code: 34149-16818, Qazvin, Iran and, School of Mathematics, Institute for Research in Fundamental Sciences (IPM),, P.O. Box 19395-5746, Tehran, Iran.

E-mail address: razaniesci.ikiu.ac.ir

\section{M.R. Mokhtarzadeh}

School of Mathematics, Institute for Research in Fundamental Sciences (IPM),, P.O. Box 193955746, Tehran, Iran.

E-mail address: mrmokhtarzadeh@ipm.ir 